\title{
Aortic dissection in port-access cardiac surgery: A safe procedure, based on team approach, protocols and TEE
}

\author{
Kennes $\mathbf{J}^{1 *}$, Coddens $\mathbf{J}^{2}$, Van Praet $\mathrm{F}^{3}$ and Delahaye $\mathbf{R}^{4}$ \\ ${ }^{1}$ Department of Anaesthesia, University Hospitals Leuven, Herestraat 49, B-3000 Leuven, Belgium \\ ${ }^{2}$ Department of Anaesthesia and Intensive Care Medicine, Onze Lieve Vrouw Clinic, Moorselbaan, 164, B-9300 Aalst, Belgium \\ ${ }^{3}$ Department of Thoracic a Cardiovascular Surgery, Onze Lieve Vrouw Clinic, Moorselbaan, 164, B-9300 Aalst, Belgium \\ ${ }^{4}$ Department Perfusionist, Onze Lieve Vrouw Clinic, Moorselbaan, 164, B-9300 Aalst, Belgium
}

\section{Introduction}

Worldwide, Port Access cardiac surgery is not routine practice. There is no single explanation for this low application. Reports about aortic dissection may explain this partially. Literature shows that the procedure is safe in the hands of experienced teams. The following case report demonstrates the importance of the team approach and the crucial role of TEE. Continued alertness of the whole team, and particularly of the anesthesiologist-echocardiographist, remains crucial in prevention of complications. This is the key for a successful program in minimally invasive cardiac surgery.

\section{Case report}

A 65-year-old female patient presented for minimally invasive repair of mitral and tricuspid valve, cryoablation for atrial fibrillation and left atrial appendage amputation, using the Port Access technique.

Her personal history included arterial hypertension, dyslipidaemia, congenital kyphoscoliosis, reflux esophagitis, atrial fibrillation and dyspnoea. She was treated with furosemide $10 \mathrm{mg}$, nebivolol $5 \mathrm{mg}$, perindopril/indapamide $5 \mathrm{mg} / 1.25 \mathrm{mg}$ and rivaroxaban $20 \mathrm{mg}$ each one single daily dose.

Biochemical analysis revealed mild renal failure and hypercholesterolemia. ECG confirmed atrial fibrillation with slow ventricular response. Cardiomegaly and kyphoscoliosis were manifest on chest radiography. Pulmonary function tests were normal.

Preoperative echocardiography showed left atrial enlargement, severe central mitral regurgitation, mitral annular dilatation and severe tricuspid insufficiency with an estimated right ventricular systolic pressure of $47 \mathrm{mmHg}+$ CVP. There was aortic valve sclerosis with mild insufficiency, normal global and regional left ventricular function with normal end-diastolic volume and concentric left ventricular hypertrophy. The coronary angiography revealed non-significant coronary wall irregularities. Angiography of the aortoiliac axis was normal.

The patient was premedicated with lorazepam $2.5 \mathrm{mg}$ orally. She was equipped with standard monitoring. A left sided $16 \mathrm{G}$ peripheral and right sided $20 \mathrm{G}$ radial arterial catheter were inserted. Anaesthesia induction and maintenance were performed using TCI (target-controlled infusion) with propofol and remifentanil at target concentrations set at 2 to $3 \mu \mathrm{g} / \mathrm{ml}$ and 2 to $3 \mathrm{ng} / \mathrm{ml}$ respectively. Antibiotic prophylaxis consisted of 4 doses of Cefazolin $2 \mathrm{~g}$ over $24 \mathrm{~h}$. Rocuronium $100 \mathrm{mg}$ was administered before insertion of a left-sided double-lumen endobronchial tube. A TEE probe was inserted, and a complete TEE exam was performed. A triple lumen central venous catheter and a venous cannula for drainage were placed via the right internal jugular vein. This cannula is connected to the extracorporeal circuit (ECC), and together with a femoral venous drainage cannula will assure venous drainage to the ECC. The authors always adhere strictly to their protocol $[1,2]$. According to this protocol, the jugular venous cannula, central venous catheter and cannula in the inferior caval vein are inserted and positioned under TEE guidance on a bicaval view. Insertion and proper position of the femoral artery cannula and the endo-aortic balloon catheter are achieved by using the long axis ascending aortic and transverse descending aortic views. Insufflation and adjustment of the position of the endo-aortic balloon is performed with TEE visualization of the long axis ascending aortic view. All cannulas and catheters are inserted using the Seldinger technique. Surgeons and anaesthesiologists adhere to the strategy of no or minimal resistance during insertion of guidewires and cannulas. In our center a coronary sinus catheter and pulmonary artery catheter are not standard of care because of the good performance of antegrade cardioplegia through the distal end of the endo-aortic balloon. The surgeon places a venting drain through the atrioventricular valve during surgery.

Via a right sided inguinal incision, after heparinization with $4 \mathrm{mg} / \mathrm{kg}$ heparin, a venous drainage cannula was advanced by the surgeon using a Seldinger technique guided on a bicaval view. Similarly, a femoral artery cannula and ascending aortic balloon catheter were passed over guidewires under TEE guidance. Under left sided one lung ventilation, a $4 \mathrm{~cm}$ right sided thoracotomy was performed. ECC was started once an ACT over $480 \mathrm{sec}$. was reached. After pericardial incision, the aorta was clamped by insufflation of the endo-aortic balloon and diastolic arrest was induced by injection of $300 \mu \mathrm{g}$ adenosine in the aortic root to facilitate precise positioning of the balloon. Myocardial protection consisted of $1000 \mathrm{ml}$ cold, crystalloid St Thomas III solution and repeated $300 \mathrm{ml}$ doses every 20 - 30 minutes. The patient was cooled to $29^{\circ} \mathrm{C}$. After left atrial incision, surgical correction included mitral valve annuloplasty (CE Physio II annuloplasty ring $30 \mathrm{~mm}$ ) and pulmonary vein isolation by cryoablation. The left atrium was closed and de-aired. Via right atriotomy a tricuspid annuloplasty (CE Physio tricuspid ring

${ }^{\star}$ Correspondence to: Jelle Kennes, Department of Anaesthesia, University Hospitals Leuven, Herestraat 49, B-3000 Leuven, Belgium, Tel: 0032472907004 ; E-mail: jelle_kennes@hotmail.com

Received: March 12, 2019; Accepted: March 26, 2019; Published: March 28, 2019 
$30 \mathrm{~mm}$ ) was performed. A temporary pacemaker lead was fixed on the left ventricle and the patient was rewarmed.

Immediately after deflation of the endo aortic balloon, the perfusionist alerted for high arterial inflow pressures and deficient ECC flow. The radial arterial pressure dropped to $15 \mathrm{mmHg}$. To exclude aortic dissection, long and transverse axis scans of the ascending and descending thoracic aorta were inspected. Spontaneous contrast in the descending aorta confirmed the low flow condition. Simultaneously the complete ECC circuit was systematically and quickly checked for any possible obstructions. No cause for the problem was detected. Within 2.5 minutes after the onset of the problem, the TEE short axis view of the descending thoracic aorta revealed a sudden re-appearance of flow on the colour flow map. Spontaneous contrast disappeared and pressure and flow conditions on ECC were normalized at that moment. TEE could not reveal any possible cause of the problem. One anaesthesiologist consistently continued his search for any possible mechanism of this short-lasting perfusion problem. On deep transgastric long and short axis views of the abdominal aorta, he found a dissection flap (Figure 1) with a large intimal tear. The dissection originated from a level not accessible by TEE but stopped below the diaphragm with a re-entry tear providing flow in both lumina (Figure 2). An initial inspection, while still on ECC, excluded major residual mitral and tricuspid regurgitation. The patient was weaned from ECC and normal antegrade blood flow was detected over the entire

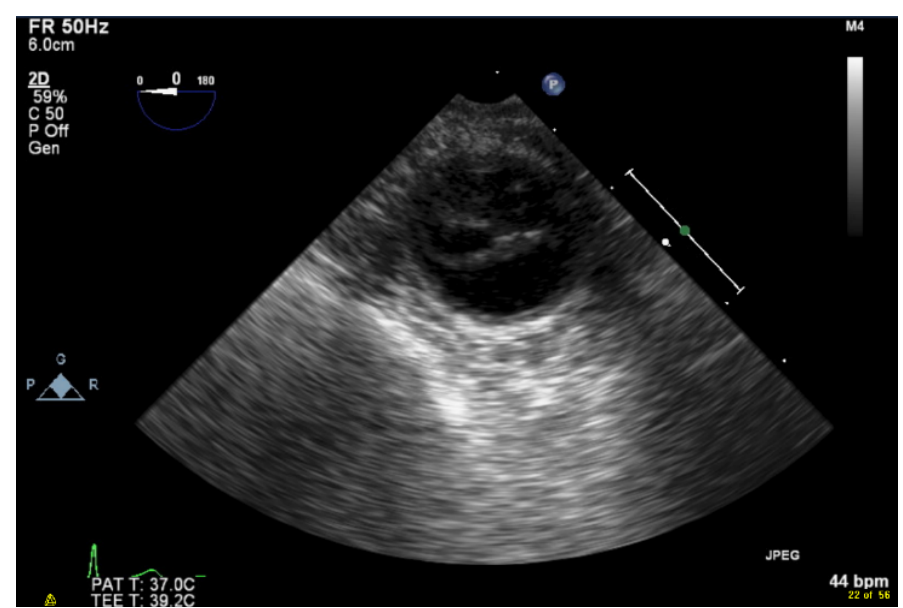

Figure 1. 2D TEE image of the descending aorta. Dissection flap and intimal tear

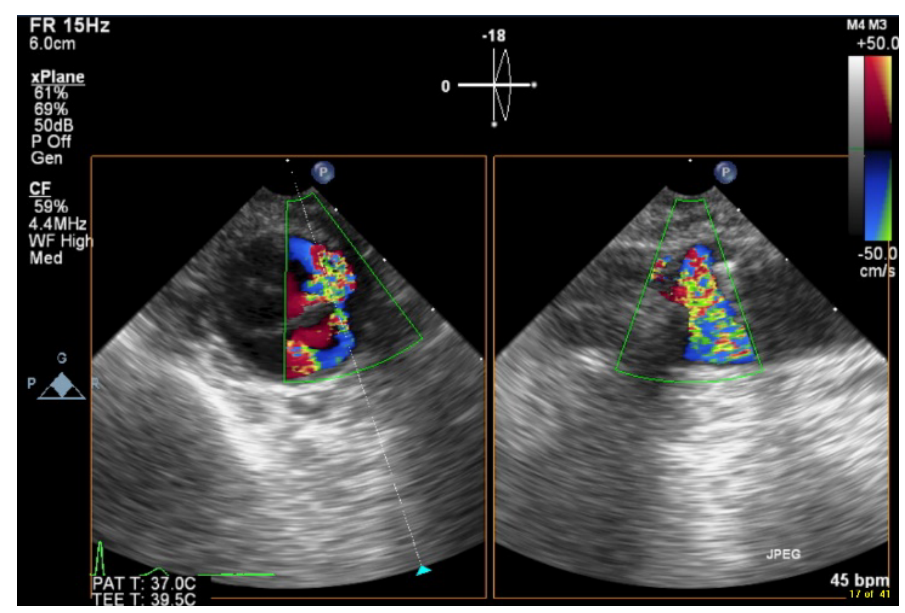

Figure 2. Color flow TEE image of the descending aorta. Communication between true and false lumen on $\mathrm{X}$ plane view aorta. Both, true and false lumina remained well perfused and a side branch of the descending aorta close to the intimal tear was seen on the colour flow map. ACT was normalized after administration of 210 mg protamine.

Bilateral ventilation was re-established after hemostasis. The incisions were closed, and the wounds were draped. The jugular cannula was removed, and the double lumen tube was exchanged for a single lumen tube. The patient was transported to the ICU with continued sedation with propofol and remifentanil.

On arrival at the ICU, a Doppler exam of both renal arteries showed bilateral renal perfusion. A postoperative CT scan of the abdominal vessels confirmed an aortic dissection from the origin of the celiac trunk to the left common iliac artery and right external iliac artery. Diuresis, lactate and arterial blood gases remained normal. A conservative treatment was preferred because no organs were endangered by the dissection. The patient is doing well one year after the operation and control CT showed a stable situation of the abdominal aorta with partially thrombosis of the false lumen.

\section{Discussion}

Port-Access cardiac surgery was first executed in 1996. Indications are coronary revascularization, mitral and tricuspid valve surgery, myxoma removal, atrial septal defect and PFO closure. Suggested advantages of minimally invasive cardiac surgery are reduced surgical trauma, reduced sternal complications, less transfusion, shorter hospital and intensive care stay, faster recovery, faster return to work and better aesthetic results [3]. Port-Access technique for reoperations offers good results because of avoiding sternal re-entry, reducing the risk of injury to patent grafts and minimizing adhesiolysis [4-7]. Furthermore, port-access offers better exposure for certain surgical procedures (e.g. mitral valve repair) [4]. Among possible disadvantages of the technique are costs [8] and concerns about the safety of PortAccess surgery. It might increase the risk for vascular complications (dissection, embolization, stroke and lower extremity ischemia) [9] and have a worse outcome if conversion to sternotomy is needed.

A meta-analysis, with its inherent limitations, by Falk et al. in 2011, suggested that the potential benefits for minimally invasive mitral valve surgery (MIMVS) might come with an increased risk of stroke, aortic dissection or aortic injury, phrenic nerve palsy, groin infections/ complications, and increased cross-clamp, cardiopulmonary bypass, and procedure time [3]. In 2015, however, results of a multicentre retrospective analysis from 10 European centers, with an experience of more than 100 procedures, by Casselman et al, was published. They reported on the 50 most recent cases of each center. They conclude that once procedural proficiency is acquired, endoaortic balloon clamping in MIMVS is a safe and effective technique, even in redo cases [10]. A retrospective study of Grossi et al. on Port-Access valve repair/ replacement shows low perioperative morbidity and mortality and comparable results to conventional sternotomy approach [11].

In the first report of the Port Access international registry in 1999 an incidence of aortic dissection of $0.75 \%$ (8/1063) was reported [12]. Generally, in cardiac surgery, the incidence of intraoperative aortic dissection ranges from $0.06 \%$ [10] to $0.35 \%$ [14]. This complication has a high morbidity and mortality up to $48 \%$ [13]. Atluri et al. [9] had a $3 \%$ aortic dissection rate in their first 100 Port-Access cases, compared with a $0.6 \%$ rate in their last 500 cases. There is an important learning curve due to the complexity of this surgical technique.

The type of aortic dissection differs between sternotomy and PortAccess. Central cannulation predisposes to type A aortic dissection, 
while both type $\mathrm{A}$ and $\mathrm{B}$ aortic dissections occur after femoral cannulation. Atluri et al. [9] compared the endo-aortic occlusion balloon with the Chitwood clamp. They found no significant difference in incidence of dissection. In the endo-aortic balloon group only type $B$ dissections occurred.

Can risk factors for aortic dissection be identified retrospectively and is prevention possible? Williams et al. [13] analyzed more than 2 million cardiac surgical cases of all types. Asian race, preoperative steroids, peripheral vascular disease and femoral cannulation were risk factors. The authors stated that there is a correlation between femoral cannulation and aortic dissection but could not establish a causeeffect relation. However, this paper was not focused on Port-Access technique.

High CPB line pressures are an alert. There are two options if an aortic dissection is detected: restoration of orthograde flow and eventual weaning from ECC or conversion to sternotomy for creation of an alternative cannulation site and surgical correction of the dissection [13]. Murphy et al. [14] identified an important difference in mortality rate between aortic dissections discovered perioperatively and those discovered 30 minutes till 21 days postoperatively: $33 \%$ and $78 \%$ respectively. This underscores the importance of perioperative active inspection with TEE to minimize end organ ischemia.

Currently, most centers use preoperative angiography, CT or MRI of the aortoiliac arteries to assess the risks of retrograde catheterization and cannulation in minimally invasive cardiac surgery, TAVI (Transcatheter Aortic Valve Implantation) and MitraClip procedures. In our case, preoperative angiography of the aortoiliac axis during cardiac catheterization was normal.

\section{Conclusion}

A review of the literature suggests that the Port Access technique is as safe as sternotomy. Our case report demonstrates how strict adherence to a protocol results in reducing complications. This is the premise for a successful program. The anaesthesiologist should be an expert in TEE. Careful TEE inspection of the ascending and descending aorta is essential at start of ECC. Any alert from the perfusionist about low flow or excessive line pressure should trigger a thorough repeated TEE exam of the aorta. The presence of spontaneous contrast, even as an isolated finding, in any part of the aorta, accessible by TEE, is to be considered as a sign of possible retrograde aortic dissection. If a dissection occurs, even if all precautionary measures were respected, the extent of retrograde aortic dissection can be limited if early recognized. Early recognition of aortic dissection and end organ dysfunction limits morbidity and mortality. Therefore, continued vigilance remains mandatory, even after years of good results.

\section{Conflicts of interest}

Dr. Van Praet is consultant and proctor for Edwards Life Sciences.

\section{Refrences}

1. Coddens J, Deloof T, Hendrickx J, Vanermen H (1999) Transesophageal echocardiography for port-access surgery. J Cardiothorac Vasc Anesth 13: 614-622 [Crossref]

2. Casselman FP, Van Slycke S, Wellens F, De Geest R, Degrieck I, et al. (2003) From classical sternotomy to truly endoscopic mitral valve surgery: a step by step procedure. Heart Lung Circ 12: 172-177. [Crossref]

3. Falk V, Cheng DC, Martin J, Diegeler A, Folliguet TA, et al, (2011) Minimally invasive versus open mitral valve surgery: a consensus statement of the international society of minimally invasive coronary surgery (ISMICS) 2010. Innovations (Phila) 6: 66-76. [Crossref]

4. Botta L, Cannata A, Bruschi G, Fratto P, Taglieri C, et al. (2013) Minimally invasive approach for redo mitral valve surgery. J Thorac Dis (Suppl 6): S686-S693 [Crossref]

5. Vallabhajosyula P, Wallen TJ, Solometo LP, Fox J, Vernick WJ, et al. (2014) Minimally invasive mitral valve surgery utilizing heart port technology. J Car Surg $29: 343-348$. [Crossref]

6. Ricci D, Pellegrini C, Aiello M, Alloni A, Cattadori B, et al. (2010) Port-access surgery as elective approach for mitral valve operation in re-do procedures. Eur J Cardiothorac Surg 37: 920-925. [Crossref]

7. Meyer SR, Szeto WY, Augoustides JG, Morris RJ, Vernick WJ, et al. (2009) Reoperative mitral valve surgery by the port access minithoracotomy approach is safe and effective. Ann Thorac Surg 87: 1426-1430. [Crossref]

8. Watson DR, Duff SB (1999) The clinical and financial impact of port-access coronary revascularisation. Eur J Cardiothorac Surg Suppl 1: 103-6. [Crossref]

9. Atluri P, Goldstone AB, Fox J, Szeto WY, Hargrove WC, et al. (2014) Port acces cardiac operations can be safely performed with either endoaortic balloon of Chitwood clamp. Ann Thorac Surg 98: 1579-1583. [Crossref]

10. Casselman F, Aramendi J, Bentala M, Candolfi P, Coppoolse R, et al. (2015) Endoaortic Clamping Does Not Increase the Risk of Stroke in Minimal Access Mitral Valve Surgery: A Multicenter Experience. Ann Thorac Surg 100: 1334-1339. [Crossref]

11. Grossi EA, Galloway AC, LaPietra A, Ribakove GH, Ursomanno P, et al. (2002) Minimally invasive mitral valve surgery: a 6-year experience with 714 patients. Ann Thorac Surg 74: 660-3. [Crossref]

12. Galloway AC, Shemin RJ, Glower DD, Boyer JH Jr, Groh MA, et al. (1999) First Report of the Port Access International Registry. Ann Thorac Surg 67: 51-58. [Crossref]

13. Williams ML, Sheng S, Gammie JS, Rankin JS, Smith PK, et al. (2010) Richard E. Clark Award. Aortic dissection as a complication of cardiac surgery: report from the Society of Thoracic Surgeons database. Ann Thorac Surg 90: 1812-1816. [Crossref]

14. Murphy DA, Craver JM, Jones EL, Bone DK, Guyton RA, et al. (1983) Recognition and management of ascending aortic dissection complicating cardiac surgical operations. $J$ Thorac Cardiovasc Surg 85: 247-56. [Crossref]

Copyright: (C2019 Kennes J. This is an open-access article distributed under the terms of the Creative Commons Attribution License, which permits unrestricted use, distribution, and reproduction in any medium, provided the original author and source are credited. 